\section{FRI0284 EFFECTIVENESS AND SAFETY OF SECUKINUMAB IN NAÏVE OR TNF-INHIBITORS FAILURE PSORIATIC ARTHRITIS PATIENTS IN REAL LIFE: A 24-MONTHS PROSPECTIVE MULTICENTER STUDY}

M. Lorenzin ${ }^{1}$, A. Carletto ${ }^{2}$, R. Foti ${ }^{3}$, M. S. Chimenti ${ }^{4}$, A. Semeraro ${ }^{5}$, L. Costa ${ }^{6}$, L. Santo ${ }^{7}$, E. Fracassi ${ }^{2}$, I. Montanari', M. Felicetti' ${ }^{1}$, G. L. Fonti ${ }^{4}$, F. Caso ${ }^{6}$, A. Doria ${ }^{1}$, A. Ortolan ${ }^{1}$, R. Ramonda ${ }^{1}{ }^{1}$ University of Padova, Padova, Italy; ${ }^{2}$ University of Verona, Verona, Italy; ${ }^{3}$ a. O.U. Policlinico Vittorio Emanuele, Catania, Italy; ${ }^{4}$ University of Rome "Tor Vergata", Roma, Italy; ${ }^{5}$ ASL Taranto, Taranto, Italy; ${ }^{6}$ University Federico II, Napoli, Italy; ${ }^{7}$ aSL BT andria - DSS4, Barletta-Andria-Trani, Italy

Background: Secukinumab (SEC) is a novel treatment for psoriatic arthritis (PsA),but data from real life are still missing.

Objectives: 1)to evaluate the effectiveness and safety of a wide cohort of PsA patients on SEC followed in 7 Italian rheumatologic centers for 24 months;2)to compare the features and disease activity indices of SEC-treated PsA patients subdivided in naïve biological drugs (group A) and in TNF-inhibitors (TNFi) failure patients (group B).

Methods: Consecutive patients with moderate-severe PsA,who begun SEC treatment were evaluated prospectively.Data on disease characteristics, previous and ongoing treatments,comorbidities and duration of follow-up were collected. Disease activity,functional and clinimetric scores and biochemical values were recorded at baseline (t0), at 6 (t6), 12 (t12), and 24 (t24) months. Anova (Kruskal Wallis) and generalized linear models were used to compare variables over time. Infections and adverse events were also collected.

Results: PsA 345 patients [38.84\% men;mean age 52.9 (11.27) years] were enrolled;mean treatment duration was 18.53 (9.97) years.SEC was prescribed as first line biologic treatment in $133(38.55 \%)$ patients and as second or more line biological treatment in $212(61.45 \%)$ patients. Enthesitis was present as a prominent manifestation in $61.44 \%$ of patients (Figure 1 ). In all population significant decrease in tender/swollen joints;Visual Analogue Scale of pain (VASp) and general health (VASgh);Psoriasis Area Severity Index (PASI);Leeds Enthesitis Index (LEI);number of dactylitis; Health Assessment Questionnaire modified for spondyloarthritis (HAQ-S);Bath Ankylosing Spondylitis Disease Activity Index (BASDAI);Bath Ankylosing Spondylitis Functional Index (BASFI);C-reactive protein (CRP) was achieved. Effectiveness of all PsA patients was associated to an improvement in Ankylosing Spondylitis disease activity score (ASDAS) $[\mathrm{t} 0=3.45(0.69)$ vs $\mathrm{t} 24=1.48$ (0.23); $<<0.01]$ and in Disease Activity in PsA (DAPSA) $[\mathrm{t} 0=29.52$ (12.56) vs $\mathrm{t} 24=11.41(7.63) ; \mathrm{p}<0.001]$.At t0 group $B$ had a more erosive $(\mathrm{p}=0.04)$ and polyarticular pattern $(p=0.04)$, a longer disease duration $(p=0.001)$, a greater prevalence $(p=0.04)$ of psoriasis and dactylitis $(p=0.01)$, a higher PASI score $(p=0.01)$, while no significant difference was observed for uveitis, inflammatory bowel diseases, enthesitis. At t 24 group A showed better physical functioning and lower disease activity compared to group $B$ [HAQs A vs $B=0.03(0.16)$ vs 0.69 (0.73); $\mathrm{BASDAI} A$ vs $B=2.37$ (0.66) vs 4.27 (2.33);ASDAS A vs $B=1.4$ (0.62) vs $1.99(0.86)$;CRP A vs $B=2.03$ (1.94) vs 3.11 (1.55) $\mathrm{mg} / \mathrm{L}$;DAPSA A vs $\mathrm{B}=7.03$ (3.57) vs 12.41 (8.05)].After t24 of treatment $74.6 \%$ of Group $\mathbf{A}$ and $72.8 \%$ of Group B articular had an inactivellow disease activity (MDA),accordingly to ASDAS and DAPSA respectively. Forty-three patients $(12.46 \%)$ stopped the treatment during the follow-up mainly because of primary or secondary loss of efficacy (29 and 24, respectively).Only 14 patients suspended SEC because of adverse events (of which 9 for reactions at site of injection).A low number of episodes of mild infections (16) occurred;SEC was instead permanently discontinued in 7 cases for:oral refractory mucositis (3);recurrent aphthosis (2);diverticulitis (2). The retention rate at t24 was good in the whole population. Interestingly no differences were found between Group A and $\mathbf{B}(\mathrm{p}=0.815)$.

Conclusion: In a real life clinical setting,SEC was safe and effective in PSA. as shown by a significant decrease of DAPSA and ASDAS over a 24-months follow up. Disclosure of Interests: Mariagrazia Lorenzin: None declared, Antonio Carletto: None declared, Rosario Foti Consultant of: lilly, sanofi, MSD, Janssen, Abbvie, BMS, celgene, roche, Speakers bureau: lilly, sanofi, MSD, Janssen, Abbvie, BMS, celgene, roche, Maria Sole Chimenti: None declared, Angelo Semeraro: None declared, Luisa Costa: None declared, Leonardo Santo: None declared, Elena Fracassi: None declared, Ilaria Montanari: None declared, Mara Felicetti: None declared, Giulia Lavinia Fonti: None declared, Francesco Caso: None declared, Andrea Doria Consultant of: GSK, Pfizer, Abbvie, Novartis, Ely Lilly, Speakers bureau: UCB pharma, GSK, Pfizer, Janssen, Abbvie, Novartis, Ely Lilly, BMS, Augusta Ortolan: None declared, Roberta Ramonda Speakers bureau: Novartis, Celgene, Janssen, Pfizer, Abbvie, Lilly

DOI: 10.1136/annrheumdis-2020-eular.3702
Baseline characteristics of 345 PSA patients of AOP treated with secukinumab in the period September 2016- November 2019

\begin{tabular}{|c|c|c|c|c|}
\hline PSA Features & Total patients & GROUPA & GROUP B & p \\
\hline Male sex $(N, \%)$ & $134(38.84 \%)$ & $52(39.09 \%)$ & $82(38.68 \%)$ & ns \\
\hline Age (vears), mean (SD) & $52.90(11.27)$ & 48.59 (13.79) & $53.30(7.99)$ & 0.05 \\
\hline Age of diagnosis (years), mean (SD) & $38.86(12.98)$ & $39.20(13.82)$ & $37.69(9.98)$ & ns \\
\hline Disease duration (years), mean (SD) & $10.68(7.64)$ & $9.18(6.82)$ & $\underline{15.76(8.32)}$ & 0.001 \\
\hline PSA (n) & 345 & 133 & 212 & NA \\
\hline Poliarticular & $209(60.58 \%)$ & $74(55.63 \%)$ & $135(63.68 \%)$ & 0.04 \\
\hline Mono/Oligoarticular & $106(30.72 \%)$ & $45(33.83 \%)$ & $61(28.7776)$ & ns \\
\hline Axial & $84(24.35 \%)$ & $28(21.05 \%)$ & $56(26.4226)$ & ns \\
\hline Enthesitis & $212(61.44 \%)$ & $81(60.91 \%)$ & $131(61.79 \%)$ & ns \\
\hline Dactylitis & $50(14.4996)$ & $13(9.7776)$ & $37(17.45 \%)$ & 0.01 \\
\hline Psoriasis, N (\%) & $247(71.59 \%)$ & $91(68.42 \%)$ & $156(73.58 \times)$ & 0.04 \\
\hline Onicopathy, $N(\mathrm{X})$ & $138(40 \% 6)$ & $47(35.34 \%)$ & $91(42.9396)$ & 0.05 \\
\hline IBD, $N(\%)$ & $4(1.15 \%)$ & $0(0 \%)$ & $4(1.89 \%)$ & ns \\
\hline Uveitis, $N(\%)$ & $14(4.06 \%)$ & $9(6.77 \%)$ & $5(2.36 \%)$ & ns \\
\hline Erosions, $\mathrm{N}(\mathrm{x})$ & $174(50.43 \%)$ & $59(44.36 \%)$ & $115(54.25 \%)$ & 0.04 \\
\hline Weight (kg), mean (SD) & $75.32(12.73)$ & $75.64(13.09)$ & $74.23(11.84)$ & ns \\
\hline Height (m), mean (SD) & $1.71(0.08)$ & $1.74(0.35)$ & $1.69(0.19)$ & ns \\
\hline BMI, mean (SD) & $25.86(3.73)$ & $25.84(3.58)$ & $25.93(4.37)$ & ns \\
\hline $\mathrm{T}[0.68]$, mean (SD) & $6.94(5.07)$ & $6.65(3.63)$ & $7.56(5.74)$ & ns \\
\hline SJ [0-66], mean (SD) & $3.77(2.67)$ & $2.28(2.87)$ & $4.21(2.16)$ & 0.04 \\
\hline LEI [0-6]mean (SD) & $1.92(1.5)$ & $181(1.56)$ & $2.14(1.48)$ & ns \\
\hline Dactylitis $[0-20]$ number digit, mean (SD) & $0.68(0.87)$ & $0.66(0.95)$ & $0.85(0.77)$ & ns \\
\hline PASI [0.72] mean (SD) & $3.21(5.59)$ & $1.05(2.25)$ & $45(6.64)$ & 0.01 \\
\hline ESR $[0.25](\mathrm{mm} / \mathrm{h})$, mean (SD) & $18.57(12.56)$ & $16.45(11.61)$ & $19.73(12.19)$ & ns \\
\hline CRP $[0-6](\mathrm{mg} / \mathrm{L})$, mean (SD) & $8.81(8.29)$ & $8.69(8.72)$ & $2.57(8.17)$ & 0.05 \\
\hline DAPSA [0-164], mean (SD) & $29.52(12.56)$ & $28.59(13.34)$ & $31.25(11.10)$ & 0.05 \\
\hline ASDAS [0-6], mean (SD) & $3.45(0.69)$ & $3.35(0.78)$ & $3.51(0.65)$ & 0.04 \\
\hline HAQ-S $[0-8]$, mean (SD) & $1.08(0.55)$ & $0.96(0.45)$ & $1.14(0.59)$ & 0.05 \\
\hline VAS-pain [0-10], mean (SD) & $6.96(1.37)$ & $6.92(1.44)$ & $7.13(1.35)$ & ns \\
\hline VAS-gh $[0-10]$, mean (SD) & $6.47(1.84)$ & $6.15(1.93)$ & $6.68(1.79)$ & ns \\
\hline BASDAI [0-10], mean (SD) & $7.02(1.54)$ & $6.62(1.44)$ & $7.23(1.51)$ & ns \\
\hline BASFI [0-10], mean (SD) & $6.43(1.94)$ & $5.77(2.08)$ & $6.82(1.78)$ & ns \\
\hline Mean treatment duration (months), mean (SD) & $17.24(11.25)$ & $16.13(10.17)$ & $18.53(9.97)$ & ns \\
\hline $1^{1 t}$ line $(N, \%)$ & $134(38.84 \%)$ & $134(100 \%)$ & $0(0 \%)$ & NA \\
\hline $2^{2 t}$ line $(N, \%)$ & $66(19.13 \%)$ & $0(066)$ & $66(31.13 \%)$ & NA \\
\hline $3^{\prime d}$ line $(\mathrm{N}, \%)$ & $67(19.42 \%)$ & $0(\infty)$ & $67(31.60 \% 6)$ & NA \\
\hline $4^{*}$ line $(N, \%)$ & $45(13.046)$ & $0(06)$ & $45(21.23 \% 6)$ & NA \\
\hline $5^{*}$ line $(N, x)$ & $15(4.3486)$ & $0(0 x)$ & $15(7.08 \%)$ & NA \\
\hline $6^{\text {th }}$ line $(N, \%)$ or more & $19(5.50 \%)$ & $0(006)$ & $19(8.96 \%)$ & NA \\
\hline Concomitant NSAIDS, N (\%) & $211(61.16 \%)$ & $95(71.43 \%)$ & $116(54.7296)$ & 0.04 \\
\hline Concomitant steroid, $\mathrm{N}(\%)$ & $95(27.54 \%)$ & $28(21.05 \%)$ & $67(31.6006)$ & 0.05 \\
\hline Concomitant CSDMARDs, $\mathrm{N}(\%)$ & $159(46.08 \%)$ & $67(50.37 \%)$ & $92(43.3996)$ & ns \\
\hline Methotrexate & $118(34.2056)$ & $51(38.35 \%)$ & $67(31.60 \%)$ & ns \\
\hline Sulphasalozine & $41(11.88 \%)$ & $18(13.53 \%)$ & $23(10.85 \%)$ & ns \\
\hline Leflunomide & $17(4.93 \%)$ & $6(4.51 \%)$ & $11(5.19 \%)$ & ns \\
\hline Hydroxychloroquine & $3(0.86 \%)$ & $2(150 \%)$ & $1(0.47 \%)$ & ns \\
\hline & & & & \\
\hline \multicolumn{5}{|c|}{ 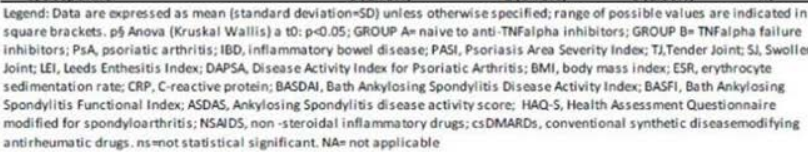 } \\
\hline
\end{tabular}

\section{FRI0285 FILGOTINIB TREATMENT RESULTS IN REDUCTION OF BIOMARKERS ASSOCIATED WITH DISEASE IN PATIENTS WITH ANKYLOSING SPONDYLITIS}

W. P. Maksymowych ${ }^{1}$, Y. Tian' ${ }^{2}$, O. K. Yoon ${ }^{2}$, W. Barchuk ${ }^{2}$, R. Galien ${ }^{3}$, R. Besuyen ${ }^{4}$, Y. Liü ${ }^{2}$, A. M. Mirza ${ }^{2}$, V. Malkov ${ }^{2}$, A. Hertz ${ }^{2}{ }^{1}$ University of Alberta, Edmonton, Canada; ${ }^{2}$ Gilead Sciences Inc., Foster City, United States of America; ${ }^{3}$ Galapagos SASU, Romainville, France; ${ }^{4}$ Galapagos BV, Leiden, Netherlands

Background: Ankylosing spondylitis (AS) is a chronic, immune-mediated disease characterized by inflammation of the sacroiliac joints and spine, and a young age of onset of 20-40 years. In the recent TORTUGA study, filgotinib (FIL), an oral, selective Janus kinase 1 (JAK1) inhibitor, significantly reduced AS disease activity compared with placebo (PBO). ${ }^{1}$ Selective JAK1 inhibition by FIL has the potential to simultaneously block multiple inflammatory pathways, thus we analyzed biomarker concentrations in serum samples from TORTUGA.

Objectives: To evaluate the impact of selective JAK1 inhibition with FIL on circulating disease associated biomarkers in adult patients with active AS enrolled in the TORTUGA study.

Methods: TORTUGA (Clinicaltrials.gov identifier NCT03117270) was a 12-week, randomized, double-blind, placebo-controlled, phase 2 study. Patients were randomized 1:1 to FIL $200 \mathrm{mg}(\mathrm{n}=58)$ or PBO $(\mathrm{n}=58)$ once-daily. Serum samples ( FIL $n=56$, PBO $n=53$ ) were collected at baseline $(B L)$ and weeks 1, 4 and 12, and analyzed using the Meso Scale Discovery immunoassay platform (Meso Scale Diagnostics, Rockville, MD, USA) to evaluate 135 biomarkers. Biomarker concentration changes from BL were analyzed on paired patient data and reported for weeks 1,4 and 12, and clustering analysis was performed. Correlation between the 135 biomarkers and selected clinical scores at BL was assessed by Spearman rank correlation analysis.

Results: FIL treatment produced significant reductions in serum concentrations of multiple biomarkers associated with AS disease activity. Five clusters of biomarker response were identified based on the kinetics and magnitude of percent changes from BL. These clusters also represented discrete biological functions: 